\title{
GESTÃO DE PROJETOS: UMA ANÁLISE CRÍTICA DA NORMA ISO 21500
}

Tiago Aparecido dos Santos, Caroline Andressa da Silva Favari, Tassiani Ayumi Nishimura, Rafael Medeiros Hespanhol.

Universidade do Oeste Paulista - UNOESTE, Curso de Engenharia de Produção, Presidente Prudente, SP. E-mail: tiago engproducao@hotmail.com.

\section{RESUMO}

O gerenciamento e direção de projetos não é uma tarefa simples para uma organização, haja vista a grande quantidade de mudanças nas questões técnicas e econômicas. A Norma ISO 21500 trata desse assunto, porém, como é um documento com menos de 10 anos, ainda surgem diversas dúvidas sobre suas aplicações. Por conta disso, o presente artigo é uma revisão de literatura que teve como objetivo descrever a Norma ISO 21500 para o gerenciamento de projetos. Para isso, os termos "certificação", "normas", "gerenciamento estratégico" e "gerenciamento de projetos" foram pesquisados diferentes bases de dados para busca de artigos originais, artigos técnicos, livros e trabalhos acadêmicos de graduação e pós-graduação em diferentes idiomas. Concluiu-se que a Norma ISO 21500 é um documento de suma importância para o gerenciamento de projetos, pois explica os métodos que devem ser aplicados e, consequentemente, potencializa o desenvolvimento da organização.

Palavras-chave: Certificação, Normas, Gerenciamento Estratégico, Gerenciamento de Projetos.

\section{PROJECT MANAGEMENT: ISO 21500 CRITICAL ANALYSIS}

\begin{abstract}
The Project management and direction is not simple task of be executed for an organization, this because of great number of changes that enterprises were submitted, especially in technical and economic aspects. ISO 21500 make some reference on this management, but how this document have less than 10 years, still occur some doubts. Then, the present study consists of a literature review that was the purpose of describe the importance of ISO 21500 for project management. For this, the words "certification", "standardization", "strategic management" and "project management" were selected and included on different databases for search of original papers, technical papers, books and academic studies of graduation and post-graduation in different languages. Conclusion indicated that Standard ISO 21500 is a document very important for project management, because it explain the methods that should be applied and consequently aid to increase the development of organization.
\end{abstract}

Keywords: Certification, Standards, Strategic Management, Project Management. 


\section{INTRODUÇÃO}

Desde a revolução industrial as organizações empresariais vêm passando por mudanças, que mexem tanto com a estrutura técnica do sistema de produção quanto com os aspectos econômicos que envolvem todos os departamentos (WINTER et al., 2006; HASS, 2007). Com estas modificações, aliadas a crise financeira mundial, o planejamento das atividades passou a ser um aspecto imprescindível para o desenvolvimento empresarial.

Além dessas questões, existe a competitividade do mercado, cada vez mais acirrada, que ocasiona a necessidade de maior preparo profissional para lidar com as mais variadas situações que podem acontecer (HASS, 2007; FERNANDEZ; FERNANDEZ, 2008). Por isso, planejar é uma forma de se preparar para enfrentar esses problemas e ainda pensar em se desenvolver.

Quando o planejamento é realizado, muitos assuntos são abordados, como, por exemplo, escopo, cronograma, custos operacionais e riscos que possivelmente incorrem em um projeto (FERNANDEZ; FERNANDEZ, 2008; COLLYER et al., 2010). Estes detalhes são extremamente importantes, pois são relativos às estratégias que podem proporcionar a melhora na qualidade dos produtos e serviços que são oferecidos pela organização, entre outras questões que podem ser abordadas (CONFORTO; AMARAL, 2010; MENA-NIETO, 2013).

Como são muitos detalhes, torna-se importante o estabelecimento de um padrão que serve como base para que as equipes de trabalho das empresas possam consultar periodicamente, como no caso da Norma ISO 21500 que é um documento que direciona o gerenciamento dos projetos (MENA-NIETO, 2013; SANJUAN; FROESE, 2013). Apesar de ser uma Norma com menos de 10 anos, sua utilização no gerenciamento de projetos vem ganhando popularidade.

Entretanto ainda existem dúvidas, especialmente quanto a sua importância e aos benefícios que podem ser obtidos com a sua utilização (SANJUAN; FROESE, 2013; KRISTINSDÓTRR; MÖLLER, 2014). Sem o devido esclarecimento, dificilmente as organizações representadas pela figura de suas equipes profissionais terão plena confiança em utilizar qualquer padrão. Dessa forma, o presente estudo se justifica pelo fato de proporcionar essas explicações, com o objetivo de descrever a importância da Norma ISO 21500 para o gerenciamento de projetos.

\section{METODOLOGIA}

O presente trabalho é uma revisão bibliográfica, na qual o tema Norma ISO 21500 foi abordado de forma descritiva, sendo que dados de outros estudos foram utilizados para a sua construção. Assim, os termos "certificação", "normas", "gerenciamento estratégico" e "gerenciamento de projetos" foram selecionados e, por conseguinte incluídos nas bases de dados Google Acadêmico e Scielo para a realização de uma busca por artigos originais, artigos técnicos, livros e trabalhos acadêmicos de graduação e pós-graduação, porém não foi estabelecido um período especifico de tempo na data de publicação dos trabalhos para que os mesmos fossem selecionados. Por sua vez, os idiomas dos estudos deveriam ser exclusivamente, Português, Espanhol e Inglês. Foram elaboradas orientações sobre gerenciamento de projetos, descrição e uso da Norma ISO 21500, tipos e execução de projetos, e segmentos usuários da ISO 21500, vantagens e desvantagens da ISO 21500, a relação da ISO 21500 com o PMBOK e as considerações finais.

\section{ORIENTAÇÕES SOBRE GERENCIAMENTO DE PROJETOS}

O gerenciamento de projetos é apontado como um padrão que pode ser seguido pelas organizações, com um conjunto de ferramentas que trabalham para aperfeiçoar as habilidades, conhecimentos e capacidades individuais, que podem contribuir com o desenvolvimento das organizações (HAS, 2007; FERNANDEZ; FERNANDEZ, 2008). Sua missão é agrupar o trabalho de várias pessoas em prol da empresa e dos clientes. 
As etapas deste gerenciamento costumam ser trabalhosas para as organizações, haja vista que planejar exige atenção aos mínimos detalhes. Adicionalmente, o foco na qualidade é uma das principais medidas, seguido pelos processos, métodos e ferramentas que são essenciais para o desenvolvimento (FERNANDEZ; FERNANDEZ, 2008; COLLYER et al., 2010). Com a descrição destes aspectos, torna-se possível direcionar as atividades e consequentemente realizar modificações de acordo com a necessidade.

Na mesma direção, os aspectos gerais podem ser contabilizados, pois já que os processos, métodos e ferramentas estão presentes, a iniciação pode ser realizada, por conseguinte as análises das possibilidades já podem ser consideradas, bem como o planejamento apresentado de forma adequada (COLLYER et al., 2010; CONFORTO; AMARAL, 2010). A definição desses passos remete diretamente a execução do que foi planejado, com a chance de inclusão de novas ideias que possam colaborar efetivamente com o desenvolvimento.

Logo adiante, isto é, com a execução, é preciso analisar a eficiência do que está sendo realizado, por isso, neste momento o procedimento mais importante é o controle, sendo assim quanto mais informações estiverem disponíveis melhor será para a organização aperfeiçoar seus métodos (CONFORTO; AMARAL, 2010; MENA-NIETO, 2013). Esta etapa é imprescindível, visto que coloca em questão a qualidade das atividades.

A última etapa se refere ao encerramento, o qual contabiliza o controle de qualidade, visto que nesta fase os erros e acertos irão aparecer detalhadamente em cada atividade que foi realizada (MENA-NIETO, 2013; SANJUAN; FROESE, 2013). A partir daí é possível corrigir as falhas e consecutivamente acrescentar novas ideias que podem ser bastante positivas para a organização, mas para isso é importante o seguinte de um padrão, como o que é definido exclusivamente na norma ISO 21500, comum à área de gerenciamento de projetos.

\section{DESCRIÇÃO E USO DA NORMA ISO 21500}

A Norma ISO 21500 foi lançada oficialmente em outubro de 2007, porém sua publicação só foi oficializada em 2012 e contou com a colaboração de 37 países, inclusive do Brasil, que teve sua participação representada pela Associação Brasileira de Normas Técnicas (ABNT) e por uma comissão especial (COLLYER et al., 2010; CCONFORTO; AMARAL, 2010). Sua criação foi uma forma de tentar reduzir as distinções de ideias que existiam entre os órgãos internacionais e os nacionais.

No que se refere ao objetivo principal, sua finalidade é direcionar o gerenciamento de projetos, principalmente pela aplicação de estratégias específicas que visam melhorar o modo como esta atividade é realizada (CONFORTO; AMARAL, 2010; DROB; ZICHIL, 2013). Desde a sua criação, as organizações têm aproveitado as informações disponíveis para se desenvolver no mercado empresarial, especialmente em tempos de crise financeira.

Este documento envolve 40 processos, em que grupos como, iniciação, planejamento, execução, controle e encerramento são descritos minuciosamente para auxiliar as organizações (DROB; ZICHIL, 2013; MENA-NIETO, 2013). Adicionalmente, ainda existe a incursão em áreas exclusivas de conhecimento como, escopo, partes interessadas, integração, recursos, tempo, assim como qualidade, risco, aquisições, comunicações e custos (SANJUAN; FROESE, 2013; BINDER; AILLAUD; SCHILLI, 2014).

Embora exista este detalhamento, esta Norma ainda não é certificada, fato que se acontecer no futuro será direcionado apenas para as organizações (BINDER; AILLAUD; SCHILLI, 2014; KRISTINSDÓTRR; MÖLLER, 2014). Mesmo assim, do momento de sua criação até a publicação oficial algumas mudanças puderam ser observadas, sendo que uma delas é a inclusão do planejamento do gerenciamento, outras são relativas ao planejamento o gerenciamento do escopo, planejamento do gerenciamento dos custos, planejar do gerenciamento do cronograma e, por último, planejamento do gerenciamento das partes interessadas (PAES, 2014). Diante disso, as organizações podem elaborar suas estratégias e enfrentar os problemas do mercado empresarial. 


\section{TIPOS E EXECUÇÃO DE PROJETOS}

De forma geral, projetos só são executados em razão das oportunidades que tendem a aparecer, sendo esta inerente a uma nova exigência legal ou uma demanda que o mercado solicita (DROB; ZICHIL, 2013; MENA-NIETO, 2013). Da mesma maneira, pode se enquadrar nesta questão, o surgimento de uma necessidade organizacional atual, que também é bastante comum.

Com relação aos tipos de projetos, existem dois em que normalmente a Norma ISO 21500 é aplicada, sendo que um deles é referente a investimentos, aplicados para alterar o modo de trabalho das organizações (MENA-NIETO, 2013; SANJUAN; FROESE, 2013). Considerados mais usuais neste modelo, o que ocorre é o fornecimento de entregas, que consequentemente podem gerar benefícios. $O$ outro é tipo relativo a projetos comerciais, que a norma denota um interesse menor, porém os mesmos não devem ser descartados, pois podem ser utilizados no mercado empresarial (SANJUAN; FROESE, 2013; BINDER; AILLAUD; SCHILLI, 2014). Neste, a forma mais comum é a oportunidade de demanda do cliente. Já a respeito dos benefícios, a produção os produtos finais, que costuma ser apontada como a principal responsável (KRISTINSDÓTRR; MÖLLER, 2014; PAES, 2014). Assim, as organizações tem que prestar atenção nos modelos e utilizar apenas aquele que se encaixa no perfil da empresa.

\section{EMPRESAS USUÁRIAS DA NORMA ISO 21500}

Com a publicação da Norma ISO 21500, algumas empresas passaram a utiliza-la, pois a mesma facilitou a forma de gerenciar os projetos, especialmente aqueles destinados a área de investimentos ou relativos ao ramo comercial (MENA-NIETO, 2013; SANJUAN; FROESE, 2013). No modelo especifico das organizações que fazem uso, as empresas de consultoria, especialmente as que possuem sede no continente europeu são as que utilizam com frequência, pois este setor parece estar mais familiarizado com a Norma (SANJUAN; FROESE, 2013; BINDER; AILLAUD; SCHILLI, 2014).

Outro ramo que costuma utilizar bastante o gerenciamento de projetos é o frigorífico, com atenção exclusiva para a capacidade de fornecimento para os clientes (PAES, 2014; VIDAL; PARDO; ARES, 2014). Em uma especificação geral, apesar do menor uso, o setor comercial também costuma gerenciar projetos. A tabela 1 indica as empresas que utilizam a ISO 21500, com lugar de sede e ramo também estão especificados.

Tabela 1. Empresas que utilizam a norma ISO 21500

\begin{tabular}{lccc}
\hline Empresa & Cidade sede & País & Ramo de trabalho \\
\hline VAE controls & Ostrava & República Tcheca & Consultoria \\
SACH Consulting & Alicante & Espanha & Consultoria \\
Grupo ACMS & Madri & Espanha & Consultoria \\
Alhambra Eidos & Albacete & Espanha & Consultoria \\
AGIPCI & Madri & Espanha & Consultoria \\
\hline
\end{tabular}

Fonte: Elaborado pelos autores

Como pode ser visto na tabela 1, as empresas que utilizam a ISO 21500 são oriundas do continente europeu, sendo que a maioria é da Espanha e realiza trabalhos de consultoria.

\section{VANTAGENS E DESVANTAGENS}

As vantagens com relação a ISO 21500 são referentes ao fato de que esta norma possui definições, características e gerenciamento do projeto, bem como programas de definição e gerenciamento, as quais são características imprescindíveis para gerenciar um projeto (MENANIETO, 2013; KRISTINSDÓTRR; MÖLLER, 2014). Mais adiante, pode ser observada também a possibilidade de realização de um projeto individual e coletivo descrevendo oportunidades e 
benefícios, além disso, existe a coleta de lições aprendidas e o controle dos recursos, entre outros (PAES, 2014; VIDAL; PARDO; ARES, 2014).

Como desvantagens, a limitação apenas à introdução dos processos, com exclusividade para entradas e saídas, fato que torna este documento com menor poder de abrangência, além da ausência de um projeto organizacional de gerenciamento, da supervisão e estratégias do projeto, dos impactos organizacionais e da não certificação (VIDAL; PARDO; ARES, 2014; TAVAN; HOSSEINI, 2016). Todavia, estas informações não desqualificam a Norma, somente alertam sobre suas deficiências.

\section{ISO 21500 e o PMBOK}

O gerenciamento de projetos é uma medida que todas as organizações devem realizar, pois ajuda a prever os riscos operacionais e econômicos, fatores importantes para o desenvolvimento de qualquer empresa (KRISTINSDÓTRR; MÖLLER, 2014; NOVY; NOVAKOVÁ; WALDHANS, 2014). Por isso, normas elaboradas com a finalidade de direcionar o modo de gerenciar tendem a ser muito proveitosas, como é o caso da ISO 21500 que foi elaborada justamente para isso e para ser aplicada tanto em todos os países.

Apesar de ainda não possuir certificação, seu uso é constante, mesmo com ferramentas similares e mais amplas, como é o caso do PMBOK, que foi lançado em 1996 e também é direcionado para o gerenciamento de projetos, porém o mesmo já é certificado e considerado uma norma padrão que apresenta mais detalhes, como, por exemplo, projeto organizacional de gerenciamento, projeto de gerenciamento de escritório, projeto de supervisão, estratégias do projeto, além de projeto de sistema de informação e o impacto organizacional (REHACEK, 2014; BRIOSO, 2015).

O que proporcionou ainda mais similaridade com a ISO 21500 foi a publicação da 5a edição do PMBOK, que gerou maior alinhamento entre os dois modelos (BRIOSO, 2015; TAVAN; HOSSEINI, 2016). Em síntese, os dois documentos tem suas qualidades e limitações, sendo critério da empresa escolher qual utilizar.

\section{CONSIDERAÇÕES FINAIS}

Com base nas informações dos estudos encontrados, pode-se concluir que o gerenciamento de projetos é uma ferramenta imprescindível para o desenvolvimento das organizações, principalmente nos aspectos operacionais e econômicos. No entanto, para gerenciar, ficou evidente a importância de normas preestabelecidas como a ISO 21500, visto que a mesma direciona os objetivos e descreve todos os passos de forma minuciosa.

Mesmo sem certificação e com limitações como, apenas a introdução dos processos, falta de um projeto organizacional de gerenciamento, bem como da supervisão e estratégias do projeto e previsão dos impactos organizacionais, a utilização desta norma pode ser benéfica para, por exemplo, um projeto organizacional de gerenciamento, supervisão e estratégias do projeto, e dos impactos organizacionais, fatores que ressaltam a significância do uso deste documento.

Seu uso é bastante requerido em vários setores, como no caso do agroindustrial que é um usuário constante da ISO 21500. Neste contexto, sua similaridade com o PMBOK não reduz sua importância. No entanto, a escolha da ferramenta de gerenciamento de projetos é uma incumbência das empresas, as quais estão conscientes de suas necessidades e possibilidades.

\section{REFERÊNCIAS}

BINDER, J.; AILLAUD, L. IV.; SCHILLI, L. The project management cocktail model: An approach for balancing agile and ISO 21500. Procedia - Social and Behavioral Sciences, v. 119, p. 182-191, 2014, https://doi.org/10.1016/i.sbspro.2014.03.022. 
BRIOSO, X. Integrating ISO 21500 Guidance on Project Management, Lean Construction and PMBOK. Procedia Engineering, v. 123, p. 76-84, 2015, https://doi.org/10.1016/i.proeng.2015.10.060.

COLLYER, S. et al. Aim, fire, aim - Project planning styles in dynamic environments. Project Management Journal, v. 41, n. 4, p. 108-121, 2010, https://doi.org/10.1002/pmj.20199.

CONFORTO, E. C.; AMARAL, D. C. Evaluating an agile method for planning and controlling innovative projects. Project Management Journal, v. 41, n. 2, p. 73-80, 2010, https://doi.org/10.1002/pmj.20089.

DROB, C.; ZICHIL, V. Overview regarding the main guidelines, standards and methodologies used in project management. Journal of Engineering Studies and Research, v. 19, n. 3, p. 26, 2013.

FERNANDEZ, D. J.; FERNANDEZ, J. D. Agile project management-agilism versus traditional approaches. Journal of Computer Information Systems, v. 49, n. 2, p. 10-17, 2008.

HASS, K. B. The blending of traditional and agile project management. PM world today, v. 9, n. 5, p. 1-8, 2007.

KRISTINSDÓTRR, L. K.; MÖLLER, E. Maximizing the Benefits of ISO 21500 Implementation. International Journal of Business and Social Science, v. 5, n. 9, p. 1, aug. 2014.

MENA-NIETO, A. UNE-ISO 21500, una oportunidad para aplicar buenas prácticas en dirección de proyectos en España. Dyna, v. 88, n. 3, p. 285-289, mayo./jun. 2013.

NOVY, M.; NOVAKOVÁ, J.; WALDHANS, M. The impact of ČSN ISO 21500 standard on project management of constructions in the Czech Republic. In: conference proceedings of People, Buildings and Environment 2014, an international scientific conference, Kroměříž, Czech Republic, p. 349-355.

PAES, L. A. B. A utilização da metodologia PMBOK no gerenciamento de projetos: uma análise dos das novas práticas propostas na 5a edição. Revista Eletrônica de Graduação do UNIVEM, Marília, n. 1, p. 170-191, 2014.

REHACEK, P. Standards ISO 21500 and PMBOK ${ }^{\circledR}$ Guide for Project Management. International Journal of Engineering Science and Innovative Technology (IJESIT), v. 3, n. 1, p. 288-295, January. 2014.

SANJUAN, A. G.; FROESE, T. The Application of project management standards and success factors to the development of a project management assessment tool. Procedia-Social and Behavioral Sciences, v. 74, p. 91-100, 2013, https://doi.org/10.1016/i.sbspro.2013.03.035.

TAVAN, F.; HOSSEINI, M. Comparison and analysis of PMBOK 2013 and ISO 21500. Journal of Project Management, v. 1, p. 27-34, 2016, https://doi.org/10.5267/j.jpm.2017.1.002.

VIDAL, J. G; PARDO, C. V.; ARES, C. A. Evaluation of the standard UNE-ISO 21500:2013 for application in agroindustrial projects. In: International Congress on Project Management and Engineering $18^{\text {th }}, 2014$, Alcañiz. Anais.... Alcañiz, 16-18th July 2014, p. 2050-2059. 
WINTER, M. et al. Directions for future research in project management: The main findings of a UK government-funded research network. International Journal of Project Management, v. 24, p. 638649, 2006, https://doi.org/10.1016/i.ijproman.2006.08.009. 